\title{
Análisis comparativo de la conducta de niños autistas, deficientes y normales en una situación de interacción
}

\section{Encarnación Sarriá *}

Universidad Nacional de Educación a Distancia

\section{Angel Rivière}

Universidad Autónoma de Madrid

\section{INTRODUCCION}

Hace ya 42 años, cuando Kanner presentó la descripción de I I niños cuyos rasgos críticos parecian constituir un único síndrome, llamado más tarde «autismo infantil precoz» (Kanner, 1943), destacó: «... lo sobresaliente, lo patognómico, la alteración fundamental, es la incapacidad para relacionarse normalmente con las personas y situaciones".

Desde entonces, los trastornos en el comportamiento social de los niños autistas se han constituido en rasgo criterial para el diagnóstico diferencial de este sindrome, y éste aparece como tal virtualmente en todas las descripciones sobre autismo encontradas en la literatura (Creak, 1964; Clancy y Rendle-Short, 1969; Reichler y Schopler, 1971; Rimland, 1971; Ornitz, 1973; Ritvo, 1976; Ritvo y Freeman, 1977). De hecho, en las escalas de diagnóstico más recientes sigue conservando su valor como rasgo criterial (v. gr., DSM III, i980; la CARS de Schopler et al., I980; la $\mathrm{ABC}$ de Krug et al., I980; la "escala para el diagnóstico de autismo» de Rivière et al., 1984).

A medida que se avanzó en el estudio del autismo y sus alteraciones asociadas, y se refinaron criterios y medios diagnósticos, la importancia atribuida a los trastornos en las relaciones sociales fue variando de un momento a otro y de un marco teórico frente a otro. $\mathrm{Y}$ aun así, a pesar de estas oscilaciones, podemos decir que nunca fue un aspecto privilegiado en la investigación sobre autismo.

Sin ser, por tanto, muy abundante la literatura sobre el tema, su heterogeneidad de objerivos, procedimientos e hipótesis explicativas es más que suficiente para dificultar de forma considerable cualquier intento de sistematización de la información que proporcionan, con la esperanza de poder clarificar los trastornos diferenciales de la conducta social de los niños autistas.

Muchas de las investigaciones que podemos encontrar al hacer una revisión sobre el tema resultan ser fundamentalmente trabajos sobre la uresponsabilidad social» de los niños autistas, en la medida en que estudian ciertos parámetros de respuesta ante ciertos estímulos sensoriales, que están relacionados de alguna forma con la sociabilidad y las situaciones de interacción (v. gr., Hutt y Ounsted, 1966, 1970; Hermelin y O'Connor, 1963 , 1970; O'Connor y Hermelin, 1967; Van DerCar et al., 1979; Uchida, 1981).

Ahora bien, incluso los estudios realizados en contextos menos artificiales y más cercanos a las situaciones reales de interacción social en las que el sujeto se desempeña normalmente (v. gr., Black et al., 1975; Richer, 1976; Rogers y Fine, 1977; Grant, 1979; Strain et al., 1976, 1979; Cantwell et al., 1979; Mc Hale, 
I983), no resuelven mucho mejor el problema. No cabe duda de que cada investigación aporta algo más en la clarificación del mismo, pero son tantas las diferencias existentes entre los estudios en cuanto a características de la muestra, los controles experimentales utilizados, las categorias de observación, el contexto de investigación..., que en conjunto no permiten grandes avances en la descripción y explicación de los trastornos específicos de este grupo patológico, siendo éste un requisito fundamental para la efectividad de la intervención terapéutica.

Con esta inquietud se diseñó una investigación que reuniría ciertos controles metodológicos que consideramos relevantes (por ejemplo, igualación en ciertas variables de los grupos a comparar), que estudiaría el comportamiento de los sujetos en situación de interacción (se escogió la interacción diádica con un adulto por las posibilidades de control que of rece), $y$ que seria ambiciosa en cuanto al número y tipo de conductas analizadas.

Se buscó un punto de equilibrio en cuanto a la posibilidad de controlar una variable que consideramos fundamental, como es el nivel de estimulación interpersonal que reciben los sujetos, y observar la actuación del sujeto en una situación con cierta semejanza a la realidad. Para ello se estableció un "guión" previo que determinaría la actuación del adulto con todos y cada uno de los niños, pero que permitiese mantener (en la medida de lo posible) cierta naturalidad.

Por otro lado, conscientes de la influencia que tiene el grado de restricción impuesto al sujeto sobre su conducta manifiesta, se realizó dicho "guión» de forma que existiesen una diversidad de situaciones graduadas en cuanto a la actividad o intrusividad del adulto sobre el niño.

Se intentó, además, preservar el significado original de las palabras «evitación y escape", en el sentido que hacen referencia a una función o efecto de la conducta, y no a un carácter intrínseco a ella misma por su peculiar topografía. De esta forma, un grupo de conductas que tradicionalmente se categorizan de evitación o escape, sólo serian registradas como tales en determinados momentos de la situación de interacción en los cuales la conducta del adulto era la causa más probable de la conducta del niño.

\section{ESTUDIO EMPIRICO}

\section{Objetivos}

Los objetivos fundamentales planteados en esta investigación son:

El estudio comparativo de la conducta de niños autistas en una situación de interacción social, a través de un análisis exhaustivo, que recoja distintos tipos de conducta teóricamente relevantes (conductas proxémicas, de orientación, de atención, expresivas, estrategias comunicativas, de evitación y/o escape, agresivas...), que en conjunto posean un valor descriptivo y puedan constituirse en potenciales variables de estudio para futuras investigaciones.

Comprobar si este análisis exhaustivo de variables conductuales, definidas por su topografía, permite diferenciar a los sujetos autistas de otros deficientes y normales cuando todos ellos están igualados en edad mental.

Comparar el nivel de conductas de "evitación y/o escape» de los sujetos autistas respecto al de los deficientes y normales, a través de su comportamiento en una situación que proporçione ciertas garantías de que no estamos interpretando equivocadamente (al imponer dicho rótulo) una serie de movimientos o conductas que podrian estar respondiendo a otro fenómeno muy distinto del de la huida o evitación de la persona y de la interacción con ella.

Y analizar, a través de los datos de dicha muestra igualada, el efecto de la variable edad mental en la conducta diferencial de los sujetos en situación de interacción social, como una primera aproximación a un interesante y prometedor tema de investigación.

A partir de dichas consideraciones se diseñó una investigación con una preocupación importante (entre otras muchas, que no lo fueron menos): que la situación de interacción, a partir de la cual se estudiaria comparativamente la conducta social de los niños consiguiese un punto de equilibrio entre el control de la cantidad, cualidad, orden y duración de la estimulación interpersonal que reciben los sujetos, y la naturalidad o su semejanza con una posible situación real. 


\section{1. Hipótesis}

- Teniendo en cuenta los resultados y las conclusiones obtenidos en estudios empiricos sobre la conducta social de los niños autistas y la reflexión crítica sobre los mismos, tratamos de elaborar unas hipótesis de trabajo, que como punto de partida recogiesen los criterios más generalizados sobre el tema y también ciertos aspectos controvertidos o polémicos sobre los cuales este estudio podría incidir con su evidencia empirica.

Por lo que indican las investigaciones y reflejan los criterios utilizados en las escalas diagnósticas, parece estar muy extendida la afirmación de que los niños autistas interactúan poco; más exactamente que, comparados con los niños normales y los deficientes no autistas, inician menos interacciones y responden de forma anómala a las que les plantean los otros (Clancy y Rendle-Short, 1969 ; Schopler, I 980 ; Richer, 1976; Strain et al., 1976; Uchida, 1981).

Recogiendo esta idea ( 1 ), predeciremos que los niños autistas presentarán un nivel significativamente menor que los sujetos deficientes y/o que los normales en cuanto a conductas de aproximación e interactivas, consideradas globalmente (2). Igualmente, presentarán un nivel significativamente menor al considerar dichas conductas aisladamente, con una excepción: lo referido a la variable proximidad, ya que son bastantes los estudios que relativizan la supuesta anomalía de la conducta de los sujetos autistas relacionada con la distancia interpersonal (v. gr., O'Connor y Hermelin, I 963 ; Hutt y Ounsted, I 970; Van DerCar et al., 1979); por tanto (3), supondremos que el grupo de sujetos autistas no se diferenciará de los otros grupos en dicha variable (proximidad).

Respecto a las conductas agresivas, son también varios los estudios que indican que los niños autistas no se caracterizan por su mayor o más frecuente conducta agresiva (v. gr. Hutt y Vaizey, I966, y Richer, I976). Por tanto (4), los sujetos autistas presentarán un nivel de conductas agresivas, consideradas globalmente, similar al de los otros grupos ( $\mathrm{s}$ ). Tampoco habrá diferencias entre los grupos al considerar aisladamente las conductas agresivas con el exterior. Sin embargo, la autoagresión está recogida por numero- sas escalas diagnósticas como un elemento caracterizador del autismo (v. gr., Freeman et al., 1979, 1980; Kruf, Arick y Almond, 1980 ); por tanto (6) suponemos que los sujetos autistas presentarán un nivel de autoagresiones mayor que los sujetos deficientes no autistas y/o que los sujetos normales.

Por otra parte, los trabajos de Richer y colaboradores (Richer, 1976; Richer y Richards, I975; Richer y Coss, 1976) forman un grupo de investigaciones ambiciosas (en cuanto al número de conductas observadas) y centradas prioritariamente en la conducta social de los niños autistas. Su principal conclusión: “los ninos autistas están predominantemente motivados a evitar los encuentros sociales» (Richer y Richards, I975, pág. 98), se reitera en cada uno de sus escritos $y$, además, coincide con una idea bastante extendida en la imagen popular sobre el niño autista. Ahora bien, aunque existen puntos en común, son bastantes los aspectos que diferencian los estudios de estos autores con el trabajo que ahora nos ocupa. Por esa misma razón sería interesante estudiar si en estas nuevas condiciones se cumplen las hipótesis de estos autores. Supondremos, entonces, que (7) los sujetos autistas presentarán un nivel significativamente mayor en las conductas de evitación $\% / 0$ escape, consideradas globalmente (8). También presentarán un nivel significativamente mayor que los deficientes no autistas $y / 0$ que los normales al considerar dichas conductas aisladamente; con una excepción: la frecuencia de comunicación verbal con contenido de evitación, pues consideramos que el déficit de lenguaje, ampliamente reconocido como característico del niño autista (vid. Rivière y Belinchón, 1981), será más determinante en la conducta lingüistica del sujeto, que su supuesta motivación al escape. Por tanto (9), los sujetos autistas presentarán una frecuencia de comunicación verbal con expresiones de rechazo y/o deseo de escape de la situación, inferior a la de los sujetos deficientes no autistas $y / 0$ normales.

\section{Método}

\subsection{Sujetos}

La muestra estaba compuesta por 30 niños de los cuales 10 fueron diagnostica- 
dos de autismo infantil precoz, 10 deficientes mentales no autistas y 10 niños normales. Los sujetos fueron igualados uno a uno, a partir de los datos del grupo de autistas, en sexo y edad mental (evaluada mediante la escala Brunet-Lézine, forma nueva). Los sujetos autistas y los deficientes no autistas fueron igualados también en edad cronológica, quedando, por tanto, igualados en cociente de desarrollo. La evaluación para la igualación de los sujetos fue realizada en todos los casos por la misma persona.

En cada grupo diagnóstico se incluyó el mismo número de niños que de niñas, ya que investigaciones recientes sobre diferencias sexuales en autismo (v. gr., Lord y cols., 1982) permiten abrigar la sospecha de su posible influencia en los resultados. Algunas de las características de la muestra se encuentran más detalladas en la Tabla I.

TABLA I

Caracteristicas de la muestra

\begin{tabular}{|c|c|c|c|c|c|c|c|c|c|}
\hline \multirow[b]{3}{*}{ Aut. } & \multicolumn{3}{|c|}{ Edad cronológica } & \multicolumn{3}{|c|}{ Edad mental } & \multicolumn{3}{|c|}{ Cociente de desarrollo } \\
\hline & $\begin{array}{c}\text { Intervalo } \\
\text { (meses) }\end{array}$ & $\mathbf{x}$ & Sx & $\begin{array}{c}\text { Intervalo } \\
\text { (meses) }\end{array}$ & $\mathbf{x}$ & Sx & Intervalo & $\mathbf{x}$ & Sx \\
\hline & $36-166$ & 70 & 29,9 & $14-54$ & 28 & I 1,9 & $18,2-\quad 7,3$ & 33,4 & 19,4 \\
\hline Def. & $29-166$ & 70 & 31,5 & $11-54$ & 28 & I 2,1 & $19-70$ & 33,6 & 19,1 \\
\hline Nor. & $15-54$ & 28 & 11,8 & $14-54$ & 28 & 11,7 & $88,6-105$ & $9^{8, \mathrm{I}}$ & 4,8 \\
\hline
\end{tabular}

Para la selección de los sujetos del grupo de autistas se realizó un primer diagnóstico clínico basado en los criterios de Rutter (1978):

a) Conductas de aislamiento o falta de desarrollo de las relaciones interpersonales.

b) Alteraciones del desarrollo del lenguaje (retraso, deterioro o falta de adquisición del lenguaje) tanto a nivel expresivo como receptivo. Presencia en los niños hablantes de rasgos específicos como la ecolalia, la inversión pronominal, literalidad.

c) Fenómenos ritualistas (insistencia en la invarianza del ambiente, manifestada en las pautas estereotipadas del juego, obsesiones y preocupaciones anormales, resistencia al cambio) y/o estereotipias motoras.

\section{d) Aparición de sintomas en los trein-} ta primeros meses de vida del niño.

Para su inclusión definitiva en la muestra experimental se les evaluó (tanto a los sujetos autistas como a los deficientes no autistas) mediante la escala de diagnóstico diferencial ABC (Autism Behavior Cheklist) de Krug, Arick y Almond (1980), que permitió una confirmación del diagnóstico por comparación con la puntuación obtenida por cada sujeto con los datos de la muestra de estandarización de dicha escala.
El grupo de sujetos deficientes no autistas se constituyó con niños que asistían a centros de educación especial, excluyendo la posibilidad de incluir aquellos que presentasen alteraciones metabóllicas (v. gr., fenilcetonuria) ya que algunos de estos trastornos se asocian con rasgos de conducta autista (Coleman, 1976). Pero aún con esta condición, se intentó formar una muestra de amplio espectro en cuanto a etiologia de la deficiencia. Quedó finalmente compuesta por sujetos diagnosticados previamente de: síndrome de Down (20 por 100), sindrome de Cornelia de Lange ( 10 por 100 ), retraso madurativo global ( 10 por 00 ) y encefalopatía de origen no determinado (60 por 100 ).

Todos los sujetos, deficientes y autistas, habían recibido tratamiento psicopedagógico, al menos durante seis meses. Ninguno de ellos asiste al centro en régimen de internado. La mayoría toman o han tomado medicación especifica.

Los diez centros de educación especial y guarderías, a los que asistían respectivamente los sujetos de la muestra, están situados en la provincia de Madrid.

\subsection{Registro de elementos conductuales}

El sistema de observación utilizado en este estudio coincide en algunos aspectos con los manejados por otros investigadores, que han proporcionado categorías de 
observación aplicables a nuestro trabajo. Pero ninguno de ellos, por si mismo, cubre por completo nuestras necesidades. Por ello, se ha construido un listado para la observación que se apoya y fundamenta en el trabajo de otros autores, y en nuestra propia experiencia, pero que incluye categorias nuevas y reformulaciones de otras más tradicionales.

Se han definido 34 categorías, que serian agrupables en tres grandes bloques: conductas de aproximación e interactivas, conductas de evitación y/o escape y conductas agresivas. Quede claro, de cualquier forma, que somos conscientes de la relativa artificialidad de dichos agrupamientos, pero esto nos posibilita un manejo de la información de utilidad en el tema que nos ocupa, y facilita la comparación de los resultados con los de otras investigaciones.

Relación de categorías de observación, con especificación de la definición operativa y el criterio de medida.

\section{Conductas de aproximación E INTERACTIVAS}

\section{Proximidad}

El sujeto se encuentra a un metro o menos de distancia del adulto, pero no hay abrazo ni contacto fisico.

Se registra el número de intervalos de diez segundos en los que aparece dicha situación. Cuando existe además contacto físico o abrazo, se registra en sus categorías específicas y sólo en ellas.

\section{Contacto fisico prolongado}

Cualquier parte del cuerpo del sujeto se encuentra en contacto con cualquier parte del cuerpo del adulto al menos durante diez segundos consecutivos.

Se registra el número de intervalos (diez segundos), en los cuales esta situación se prolonga durante todo el intervalo.

\section{Contacto fisico activo}

Toca al adulto con la/s mano/s o con cualquier otra parte su cuerpo.
Se contabiliza el número de veces que se presenta dicha conducta.

\section{Desplazamientos hacia}

Avanza en dirección al adulto por lo menos un cuadrado $(0,6 \mathrm{~m} . \times 0,6 \mathrm{~m}$.)

Se contabiliza de forma acumulada el número de cuadrados recorridos en los desplazamientos hacia el adulto.

\section{Se vuelve bacia}

Gira todo el cuerpo o sólo el tronco en dirección al adulto.

Se contabiliza el número de veces que se presenta dicha conducta.

\section{Vuelve la cabeza hacia}

Gira la cabeza, pero no el tronco en dirección al adulto.

Se contabiliza el número de veces que se presenta dicha conducta.

\section{Mira al}

Mira al adulto, a cualquier parte de su cuerpo, excepto a su cara.

Se contabiliza el número de veces que se presenta dicha conducta.

\section{Mira a la cara (global)}

Mira a la cara del adulto.

Se contabiliza el número de veces que se presenta dicha conducta.

\section{Mira a la cara (sólo)}

Mira a la cara del adulto y no realiza simultáneamente ninguna conducta expresiva (sonrisa, llanto, abrazo) ni comunicativa (señala, tira de, comunicación verbal, vocalizaciones, muestra/da y reproduce).

Se contabiliza el número de veces que se presenta dicha conducta.

\section{Co-orientación visual}

Mira el mismo objeto o el mismo punto que en ese momento está mirando el adulto. 
Se contabiliza el número de intervalos de diez segundos en los que se presenta dicha conducta.

Esta categoría se utilizó sólo en tres minutos concretos de la situación de interacción (minutos ocho, nueve y diez), cuyas caracteristicas facilitan la fiabilidad de la medida.

\section{Sonrisa}

Sonríe y, al menos en algún momento de la sonrisa, mira a la cara del adulto.

Se contabiliza el número de veces que presenta dicha conducta.

\section{Abrazo}

Rodea el cuello o el tronco del adulto con uno o ambos brazos, sin lastimarle.

Se contabiliza el número de veces que presenta dicha conducta.

\section{Tira de:}

Tira del adulto para conseguir que haga algo que él desea o bien para mostrarle algo o a alguien.

Se contabiliza el número de veces que se presenta dicha conducta.

\section{Reproduce:}

Reproduce, dirigiéndose al adulto, parte de una acción determinada.

Se contabiliza el número de veces que se presenta dicha conducta.

\section{Comunicación verbal:}

Emite frases completas o palabras significativas, cuyo contenido no es de evitación y/o rechazo.

Se contabiliza el número de emisiones significativas, cualquiera que sea su longitud.

\section{Señala:}

Extiende y orienta en una dirección específica el/los brazos o el dedo índice.
Se contabiliza el número de veces que se presenta dicha conducta.

\section{Muestralda:}

Lleva un objeto hasta el adulto y se lo muestra, lo deja muy cerca de él o se lo entrega poniéndoselo en la/s mano/s o en la/s pierna/s.

Se contabiliza el número de veces que se da cualquiera de esas conductas descritas.

\section{Vocalizaciones:}

Emite vocalizaciones y mira a la cara del adulto al menos en algún momento de la secuencia de emisión.

Se contabiliza el número de secuencias de emisión dirigidas al adulto, tomando como criterio la mirada a la cara del adulto.

\section{Coge:}

Coge el objeto que le ofrece o le entrega el adulto.

Se registra la ausencia o presencia de esta conducta por parte del sujeto, en el tercer minuto de la situación de interacción (único ofrecimiento realizado por el adulto a todos los sujetos).

\section{Acude llamada:}

Atiende a la demanda del adulto y la ejecuta, iniciándola a los diez segundos inmediatos a su emisión o antes.

Se contabiliza la ausencia o presencia de esta conducta en dos ocasiones concretas de la situación de interacción (en el último minuto de la sesión).

\section{Conductas de evitación Y/O ESCAPE}

\section{Se aleja:}

Ante los intentos de interacción del adulto el sujeto se desplaza en una dirección concreta, alejándose del adulto, y avanza en esa dirección al menos un cuadrado $(0,6 \times 0,6$ metros). 
Se contabiliza de forma acumulada el número de cuadrados recorridos en cada uno de estos desplazamientos, que se produjesen durante los tiempos denominados activos de la situación de interacción.

\section{Se vuelve de:}

Ante los intentos de interacción del adulto, el sujeto gira el cuerpo o sólo el tronco, presentando la espalda o el perfil al adulto.

Se contabiliza el número de veces que se presenta dicha conducta, durante los momentos considerados «activos» de la situación de interacción.

\section{Vuelve la cabeza de:}

Ante los intentos de interacción del adulto, el sujeto gira solamente la cabeza, o la baja, retirando su cara de la posición de frente a la del adulto.

Se contabiliza el número de veces que tiene lugar dicha conducta, durante los momentos denominados «activos».

\section{Cara a la pared:}

El sujeto se encuentra a un metro o menos de la pared y cara a ella, al menos durante cinco segundos consecutivos, y no hay evidencia de que esté explorando algo.

Se contabiliza el número de intervalos de diez segundos en los que tiene lugar dicha conducta.

\section{Cabeza baja}

El sujeto mantiene la cabeza baja, llevando la barbilla hacia el pecho (puede presentar inclinación hacia uno de los lados), al menos durante cinco segundos consecutivos. No hay evidencia alguna de que el niño esté explorando algo.

Se contabiliza el número de intervalos de diez segundos en los que se presente dicha conducta.

\section{Llantos $y / 0$ gritos}

El sujeto llora o lloriquea, con o sin lágrimas $y / 0$ emite sonidos $y / 0$ emite vocalizaciones de volumen elevado y desagradables.

Se contabiliza el número de intervalos de diez segundos en los que aparece dicha conducta.

\section{Aparta al adulto}

Ante los intentos de interacción del adulto, el sujeto aparta con su/s mano/s, $\mathrm{su} / \mathrm{s}$ brazo/s, su/s pie/s... (cualquier parte del cuerpo utilizada activamente), al adulto o a una parte del cuerpo de éste (por ejemplo, la mano que le acaricia o interviene en sus materiales).

Se contabiliza el número de veces que aparece dicha conducta en los momentos considerados «activos".

\section{Cierra los ojos}

Ante los intentos de interacción del adulto, el niño cierra fuertemente los ojos o parpadea de forma exagerada.

Se contabiliza el número de veces que presenta dicha conducta durante los momentos denominados «activos».

\section{Se tapa los ojos y lo los oidos}

Ante los intentos de interacción del adulto, el sujeto se tapa un/los ojo/s y/o un/los oido/s con una o ambas manos o con uno o ambos brazos.

Se contabiliza el número de veces que se presenta dicha conducta durante los momentos considerados "activos» de la situación de interacción.

\section{Se protege}

Ante los intentos de interacción del adulto, el sujeto se protege cubriéndose la cabeza o el cuerpo o con uno o ambos brazos, o encogiéndose de forma repentina.

Se contabiliza el número de veces que presenta dicha conducta durante los momentos denominados "activos" de la situación de interacción. 
Emite frases completas o palabras significativas, cuyo contenido es de evitación y/o rechazo.

Se contabiliza el número de emisiones significativas, cualquiera que sea su longitud.

\section{Conductas agresivas}

\section{Agresiones al entorno (destructivas)}

El sujeto arroja con fuerza, golpea, pisotea, da patadas, muerde... los juguetes, los muebles, la puerta u otros elementos no vivos del entorno.

Se contabiliza el número de veces que se presenta cualquiera de las conductas recogidas en esta categoría.

\section{Agresiones al adulto}

El sujeto golpea, araña, da patadas, muerde, empuja bruscamente... al adulto.

Se contabiliza el número de veces que presenta cualquiera de las conductas recogidas en esta categoría.

\section{Autoagresiones}

El sujeto se muerde, se araña, se tira de los pelos, o se golpea con o contra objetos.

Se contabiliza el número de veces que se presenta alguna de las conductas recogidas en esta categoría.

\subsection{Procedimiento}

\subsubsection{Recogida de información: sesión de interacción social}

Todos los sujetos fueron grabados mediante vídeo en una sesión de 20 minutos, siendo estas filmaciones el material de observación para el estudio de las variables dependientes.

Esta sesión consistía en una situación de interacción diádica con un adulto (el mismo para todos los sujetos, mujer, de 23 años, licenciada en Psicología y con experiencia en el trato y educación de niños autistas deficientes y también normales en edad preescolar). Ios sujetos conocían al adulto de la sesión de evaluación de edad mental y sólo de eso; de esta forma podríamos considerarlo como una persona "casi» extraña.

Además del adulto y el niño, se encontraba en la sala el operador de la cámara. Tanto el aparato como la persona que lo manejaba eran visibles para el niño. Aunque esto podría causar algunos efectos no deseables, se consideró prioritario obtener la precisión de imagen necesaria para poder conseguir la información deseada. De cualquier forma, el operador se encontraba situado en un rincón (en diagonal con la posición "prefijada" del adulto) y con un espacio de movimientos delimitado.

El adulto tenía prefijada una posición a la que se dirigía en determinados momentos de la sesión de interacción. El punto elegido en cada sala estaba siempre en un rincón, de tal forma que dejaba un cuadrado $(0,6 \times 0,6$ metros $)$ de distancia a cada una de las paredes.

El sujeto era llevado a una sala del centro, al que acude regularmente, por una persona de la plantilla del mismo (director o educador) y, por tanto, conocida para el niño. Esta persona presentaba al niño, la mujer con la que iba a jugar y el operador de la cámara; ambos trataban al niño de manera informal, durante breves minutos, con el fin de suavizar la impresión; después, la mujer y el niño, avanzaban juntos hasta la posición "prefijada» del adulto, y de esta forma la sesión siempre se iniciaba en posición de proximidad entre ellos.

En dicha sesión, el adulto actuaba según un "guión social» predeterminado, con el fin de asegurar la posibilidad de control de la cantidad, cualidad, orden y duración de la estimulación interpersonal que reciben los sujetos. Este guión se diseñó con gran preocupación por salvar la naturalidad de la situación (dentro de unos límites) y por ello la actuación del sujeto tiene carácter de intrusión progresiva. La información de los distintos momentos de la sesión $\mathrm{y}$, por tanto, de cambios en su comportamiento, se le proporcionaba al adulto por un sonido peculiar emitido por un magnetófono presente en la sala. 
Con el fin de garantizar la constancia de su comportamiento de unos sujetos a otros, el adulto realizó un entrenamiento previo con niños no pertenecientes a la muestra experimental, pero de características similares. Las sesiones de entrenamiento se grabaron en vídeo, permitiendo el automoldeado de la conducta hasta conseguir su ajuste a los criterios establecidos.

Este esquema de actuación (guión social) fue el siguiente: dad.

Se parte de una situación de proximi-

\section{I.er sonido: (minuto o)}

No hay materiales lúdicos en la sala.

El adulto saluda al sujeto diciendo: "Hola, yo me llamo..., vamos a estar un rato juntos.» Continúa mirando la cara del niño en actitud pasiva.

\section{$20^{\circ}$ sonido: (minuto I) (sin materiales)}

Baja la vista y se mira las manos, levantándola a los ro segundos (atento a las señales de intervalo que propociona el cronómetro), después de otros 10 segundos vuelve a bajarla, y en otros 10 segundos volverá a levantar la vista buscando la cara del sujeto, y así sucesivamente.

\section{3.er sonido: (minuto 2) (sin materiales)}

Llama la atención del sujeto diciendo su nombre (y si éste se encuentra lejos se levanta y se acerca amablemente a él), le ofrece un caramelo, tendiendo la mano hacia él y manteniéndola a una distancia aproximada de $20 \mathrm{~cm}$. del pecho del sujeto, un máximo de ro segundos; finalizados éstos, si aún el sujeto no lo ha cogido, lo deja cerca de él. Acto seguido, acaricia al sujeto en los brazos, la cabeza y la cara hasta escuchar el siguiente sonido. Acompaña esto de verbalizaciones positivas.

\section{$4 .^{\circ}$ sonido: (minuto 3) (sin materiales)}

Deja de acariciar al niño y permanece en ese punto en actitud pasiva hasta el siguiente sonido.
5. ${ }^{\circ}$ sonido: (minuto 4) (sin materiales)

Vuelve a su posición inicial (previamente determinada) y sentándose en el suelo, mantiene alli una actitud pasiva.

\section{6. ${ }^{\circ}$ sonido: (minuto 8) (con materiales)}

Se levanta, coge la bolsa de materiales situada detrás de ella y se acerca al sujeto. Llama su atención mirándole y dice: «Mira cuántos juguetes.) Se sienta y manipula los juguetes, sin tratar de llamar la atención del niño sobre ellos. El tipo de actividades que realiza son: modelar plastilina, construcciones con cubos (torre y tren), dibujar...

\section{7. ${ }^{\circ}$ sonido: (minuto II) (con materiales)}

Comienza una especie de juego entregando todos los materiales al sujeto, colocándoselos por encima, cogiéndole algunos, cambiándolos de posición. Utiliza verbalizaciones expresivas y positivas que acompañan la acción.

\section{8. ${ }^{\circ}$ sonido: (minuto 13 ) (con materiales)}

Retira los materiales de forma no brusca y comienza a realizar juegos corporales con el niño: cosquillas, darle vueltas por el aire...

\section{9. ${ }^{\circ}$ sonido: (minuto Is) (con materiales)}

Se aleja con suavidad del sujeto y se coloca en su posición inicial dićiendo: "Yo me voy a mi sitio...», y permanece allí en actitud pasiva hasta escuchar el siguiente sonido.

\section{0. sonido: (ninuto I9) (con materiales)}

Llama al sujeto por su nombre diciendo: «... ven aquí», y apoyando la consigna con gestos. Acto seguido, pide la colaboración del sujeto para guardar los juguetes en la bolsa, apoyando gestualmente también esta consigna.

\section{It. sonido: (minuto 20) (sin materiales)}

Finaliza la grabación del vídeo y el adulto se despide del niño. 
Convendría aclarar que lo que denominamos actitud pasiva consiste en que el adulto permanece atento a la cara y movimientos del sujeto, respondiendo de forma discreta a sus intentos de interacción, pero no inicia o provoca interacciones, ni se empeña en prolongarlas. El adulto responde con interés a las llamadas de atención o demandas que le haga el sujeto, siempre que no supongan un cambio sustancial en las condiciones que acabamos de describir.

Como se puede observar en la descripción de la sesión, se plantean situaciones con material lúdico disponible para el uso del niño y otras sin él. Su objetivo es obtener una información más completa, ya que en determinados niveles de desarrollo los materiales suponen un gran apoyo (como intermediarios) en las situaciones de interacción social.

Con ese mismo objetivo se introducen momentos pasivos, en los cuales podremos estudiar la conducta más espontánea, $\mathrm{y}$ otros en los cuales es el adulto quien controla y proporciona las claves de la situación.

\subsubsection{Aparatos, lugar y materiales}

Para la grabación de las sesiones de interacción se utilizó un vídeo de media pulgada Betamax F-I marca Sony. En concreto, magnetoscopio SL, F I E y cámara HVC $4000 \mathrm{P}$ (Trinicon). La cámara llevaba adosado a uno de sus lados, y en proximidad al micrófono multidireccional un cronómetro digital Casio CartTime ST $24 \mathrm{~F}$, que emitía cada ro segundos un sonido brevísimo, grabándosé en la banda sonora de la película. Magnetófono, marca Sanyo, modelo ıoo $\mathrm{A}$, que contenía una cinta grabada con una secuencia determinada de sonidos que informaban al adulto de la duración (principio y final) de los diferentes momentos de la situación de interacción. Condiciones lumínicas propias del medio video, $5.300^{\circ}$ Kelvin, dos antorchas de 1.000 vatios.

Las grabaciones se realizaron en salas de los centros a los que asisten regularmente los sujetos experimentales. Sus dimensiones oscilaban entre $2,5 \times 3$ metros y $3,5 \times 4,5$ metros. Previamente a la filmación, la sala se vaciaba. de muebles. El negra, en cuadros $(0,6 \times 0,6$ metros $)$, facilitando asi, en el análisis posterior de las películas, la codificación de variables sobre desplazamientos motores y distancia interpersonal.

Aunque no fue posible la utilización de una sala única para los 30 sujetos (como hubiera sido deseable) se procuró que ninguno de los grupos diagnósticos realizase sistemáticamente la sesión de interacción en el mismo tipo de sala (grande, mediana o pequeña). De esta forma podemos considerar que las diferencias en cuanto al tamaño de la sala afectan de forma similar a los tres grupos, por lo cual no creemos que este factor pueda haber influido de forma significativa en nuestros resultados.

Respecto a los materiales lúdicos que habrían de ser utilizados en la sesión de interacción, se eligió una gama con distintos niveles de manipulación y complejidad, dado el amplio intervalo de edad mental de la muestra (de 14 meses a cuatro años y medio):

- Io cubos de construcción de colores en plástico.

- Juego de 8 cubos apilables en disminución de tamaño.

- Dos folios de papel en blanco y pinturas de cera.

- Plastilina blanca.

- Un muñeco de goma pequeño.

- Un gato de peluche, tamaño mediano.

- Una muñeca de trapo, también tamaño mediano.

\subsubsection{Codificación de la información}

La transformación de la información potencial de las filmaciones en datos numéricos se realizó mediante el visionado de las peliculas y el registro de las categorías de observación ya descritas, de acuerdo a sus criterios de medida específi$\cos$.

Dos personas, de forma independiente, fueron responsables de esta tarea, en todas y cada una de las grabaciones. Se realizó un período de entrenamiento, con películas de sujetos ajenos a la muestra experimental, durante el cual se discutie- 
ron y unificaron criterios atendiendo a las definiciones operativas de las categrorias. Finalizado éste no había intercambio de información sobre la codificación.

El orden de presentación de las películas fue aleatorio. Aunque el conocimiento previo de la pertenencia de los sujetos a uno u otro grupo diagnóstico era inevitable para uno de los observadores, el otro no recibió ninguna información sobre el tema.

Al estar las grabaciones divididas, en su tiempo total, en intervalos de ro segundos, la estrategia utilizada fue el análisis de cada intervalo, reponiéndolo cada vez que fuera necesario, con el fin de seleccionar el foco de atención en cada repaso; y de esta forma la codificación de los datos se realizó de forma controlada, $\sin$ riesgo de solapar información.

Los observadores manejaban dos tipos de registro, alternando su uso según fuese considerado "activo" o "pasivo" el momento de la grabación. Dicho con otras palabras, según se habia juzgado «a priorin, en qué momentos la conducta del adulto era la causa más probable de la conducta del niño (momento "activo") o no (momento "pasivo").

La hoja de registro utilizada para los momentos uactivos» incluía las 34 categorias de observación ya comentadas. I a utilizada para los momentos "pasivos" sólo 27; no se consideraban siete categorías con carácter de evitación activa $y / 0$ escape (se aleja, se vuelve de, vuclve la cabeza de, aparta al adulto, cierra los ojos, se tapa los ojos/oídos, se protege).

I os datos de las dos hojas de registro, por sujeto, se sumaron de tal forma que los datos tratados estadísticamente son una puntuación única para cada categoría de observación, para cada sujeto.

I a distribución de la sesión de veinte minutos en "activos» o "pasivos" responde al siguiente esquema:

$$
\begin{array}{r}
\text { 1.er sonido: (minuto o) } \\
20 \text { segundos, activo. } \\
40 \text { segundos, pasivo. }
\end{array}
$$

2. ${ }^{\circ}$ sonido: (minuto I)

I minuto, pasivo.

3 er $^{\mathrm{en}}$ sonido: (minuto 2)

I minuto, activo. $44^{\circ}$ sonido: (minuto 3 )

I minuto, pasivo.

s. sonido: (minuto 4 )

4 minutos, pasivo.

6. ${ }^{\circ}$ sonido: (minuto 8 )

20 segundos, activo.

2 minutos y 40 segundos, pasivo.

$7 .^{\circ}$ sonido: (minuto I I)

2 minutos, activo.

8. ${ }^{\circ}$ sonido: (minuto I 3 )

2 minutos, activo.

$9 .^{\circ}$ sonido: (minuto Is)

4 minutos, pasivo.

Io. sonido: (minuto 19)

I minuto, activo.

\section{Resultados y discusión}

Un primer dato a destacar es que, aplicado el coeficiente de Spearman a los dos grupos de datos obtenidos por los observadores, los coeficientes de fiabilidad presentan una media de 0,984 , siendo su recorrido de 75 a 1 .

Otro aspecto está relacionado con uno de nuestros principales empeños: el intento de control de la variable estimulación interpersonal que reciben los sujetos $y$ su conciliación con la naturalidad de la situación. I a forma de control elegida es que el adulto se comporte sistemáticamente igual con cada uno de los sujetos. Para comprobarlo, dos jueces estimaron la adecuación de la conducta del adulto a los criterios que habian sido descritos «a priori», valorándola en una escala de cero a diez. Visionaron las grabaciones de is sujetos (so por ıoo), cinco de cada grupo diagnóstico, elegidas aleatoriamente. Tras una prueba con un sujeto ajeno a la muestra, con discusión y comentario de los criterios, comenzaron su tarea de forma independiente. Las puntuaciones oscilan entre 8,5 y 10 , siendo la media total 9,3 , lo que permite afirmar el ajuste de la actuación del adulto a los criterios establecidos, más aún siendo 0,995 el coeficiente de fiabilidad conjunta de los jueces.

\subsection{Resultados y discusión: Parte I}

Tras las comprobaciones previas para la elección del estadístico adecuado, se 
aplicó Anova paramétrico para las variables que presentaron las características adecuadas para este tipo de análisis y no paramétrico en las restantes, pasando posteriormente al uso de la prueba de Scheffé o la U de Mann-Whitney, en su caso, para la comprobación de la significación entre cada par de grupos.

En la preparación (suma) de los datos para el análisis de los tres grandes bloques de conducta, se excluyó la variable «Mira a la cara (sólo)" por no utilizar dos veces los datos sobre contacto visual, permaneciendo la variable «mira a la cara (global)».

Los resultados se especifican en las tablas II, III, IV y V.

\section{TABIA II}

Aplicación de Anova paramétrico de un criterio de clasificacion para tres grupos: autistas, deficientes y normales.

\begin{tabular}{ccc}
\hline Variable & Estad. F & N.S. \\
\hline
\end{tabular}

Proximidad …..... 0,6084 .5919

Desplazamientos hacia $\ldots 1,7692 \quad .1894$

Se vuelve hacia ....... 0,1105 .8958

Vuelve la cabeza hacia . 3,1995 .0566

Mira a la cara (sólo) ....3,7603 .0363 *

Mira a la cara (global) ... 2,4096 .1089

Coordinación visual $\ldots 3,3775 \quad .0491$ *

Se aleja ........... 2,6772 $\quad 0872$

Se vuelve de ....... 0,7325 49900

Vuelve la cabeza de .... $0,0429 \quad .9581$

Aparta al adulto ...... 0,6821 .5141

* p. $(\alpha) \leqslant .05$.

\section{TABLA III}

Aplicación de la prueba de Scheffé para el análisis de la significación de cada par de grupos.

\begin{tabular}{|c|c|c|c|}
\hline Variable & Aut-Nor. & Aut-Def. & Def-Nor. \\
\hline $\begin{array}{r}\text { Mira a la cara } \\
\text { (sólo) . . . } \ldots \\
\text { Coorientación } \\
\text { visual ...... }\end{array}$ & $\begin{array}{l}2,7410^{*} \\
2,5984^{*}\end{array}$ & $\begin{array}{l}1,4437 \\
1,2528\end{array}$ & 1,2970 \\
\hline
\end{tabular}

Para las variables señaladas con ${ }^{*}$ podemos afirmar que existen diferencias significativas entre esos dos grupos de sujetos.

* Estadístico 2,59.
TABI.A IV

Aplicación de Anova no paramétrico (Kruskal-Wallis) de un criterio de clasificación por rangos para tres grupos

\begin{tabular}{rrr}
\hline Variable & Estad. H. & N.S. \\
\hline
\end{tabular}

Contacto físico pasivo $\ldots \quad 2,2664 \quad .3220$

Contacto fisico activo . . . 6,6680 $\quad .0356$ *

Mira al ............ 3,2091 .2010

Sonrisa ............ 2,0659 .3559

Abrazo ............ 0,2513 .8819

Tira de ............ $0,9823 \quad .6119$

Reproduce ........... 1,3109 .5192

Comunicación verbal ... $1,3109 \quad, 5192$

Señala . . . . . . . . 2,4252 .2974

Muestra/Da ........ 4,6794 .0966

Vocalizaciones ........ $0,3754 \quad .8289$

Coge ............. 1,2083 .5469

Acude llamada . . . . . . . $0,9942 \quad .6083$

Cara a la pared ........ $0,9638 \quad .617^{6}$

Cabeza baja .......... 2,2190 .3296

Llantos/gritos ........ $0,6531 \quad .7214$

Cierra los ojos ........ 4,1129 .1279

Se tapa ojos/oidos ...... $5,1148 \quad .0775$

Se protege ......... 4,7046 .0951

Comunicación verbal (E) , 1,0286 .9979

$\begin{array}{lll}\text { Agresiones al entorno .... 5, } 51148 \quad .0775 & \end{array}$

$\begin{array}{llll}\text { Agresiones al adulto } \ldots . .4 & 4,9200 & .0894\end{array}$

Autoagresiones .........

$\begin{array}{lll}\text { Conductas de aproximación } 5,5339 & .0628\end{array}$

e interactivas (global) ..

Conductas de evitación $y / 0 \quad 7,6067 \quad .0223$ *

escape (global) ........

$\begin{array}{lll}\text { Conductas agresivas } & 2,0243 \quad .3634\end{array}$

(global) ............

$4,5113 \quad .1048$

* p. $(x) \leqslant .09$.

Como podemos observar, al analizar los datos de las variables aisladamente:

Los sujetos autistas presentan una frecuencia de miradas a la cara del adulto (no asociadas a otra conducta expresiva o comunicativa) y de coorientación visual con el mismo, significativamente menor que los sujetos normales. El nivel de los sujetos autistas en ambas variables es también inferior al de los sujetos deficientes no autistas, pero no lo suficiente para que la diferencia resulte estadisticamente significativa [por tanto, la hipótesis (2) se cumple, aunque sólo sea parcialmente].

Los sujetos deficientes no autistas presentan una frecuencia de contactos físicos activos significativamente mayor que el grupo de normales.

No aparecen diferencias estadisticamente significativas entre los grupos en las 31 
Aplicación de la prueba U de Mann-Whitney para la comprobación de la significación entre cada par de grupos.

\begin{tabular}{|c|c|c|c|c|c|c|}
\hline \multirow[b]{2}{*}{ Variable } & \multicolumn{2}{|c|}{ Aut-Nor. } & \multicolumn{2}{|c|}{ Aut-Def. } & \multicolumn{2}{|c|}{ Def-Nor. } \\
\hline & $\begin{array}{l}\text { Estadistico } \\
\text { empirico }\end{array}$ & N.S. & $\begin{array}{l}\text { Estadistico } \\
\text { empirico }\end{array}$ & N.S. & $\begin{array}{l}\text { Estadistico } \\
\text { empirico }\end{array}$ & N.S. \\
\hline $\begin{array}{l}\text { Contacto físico activo } \ldots \ldots \ldots \\
\text { Conductas de aproximación e inte }\end{array}$ & 23 & .0204 & 48 & .4546 & $8 I$ & $.0085^{*}$ \\
\hline racción $\ldots \ldots \ldots \ldots$ & 22 & .0171 & 16 & $.00 \mathrm{~s}^{*}$ & 55 & .3527 \\
\hline
\end{tabular}

* p. $(\alpha) \leqslant 02$.

variables restantes (por tanto, se cumplen las hipótesis 3 y 5 y no se cumplen las 6 , 8 y 9).

$\mathrm{Al}$ agrupar las variables en tres grandes bloques:

No aparecen diferencias estadísticamente significativas entre los grupos respecto a las conductas de evitación y/o escape (por tanto, no se cumple la hipótesis 7).

Tampoco aparecen diferencias estadísticamente significativas entre los grupos respecto a las conductas agresivas (por tanto, se cumple la hipótesis 4).

Sin embargo, en cuanto a las conductas de aproximación e interactivas, los sujetos autistas presentan un nivel significativamente inferior que los sujetos normales y que los deficientes no autistas (por tanto, se cumple la hipótesis i).

Antes de comentar en profundidad la significación teórica de los resultados obtenidos, creemos indispensable hacer referencia a algunas limitaciones que posee este estudio y que necesariamente nos obligan a aumentar nuestra cautela en la interpretación de los datos. Las señalaremos esquemáticamente.

Todos los sujetos conocían (de la evaluación psicométrica) al adulto con el que iban a interactuar. Asimismo, la situación de interacción era nueva para todos los sujetos. Ahora bien, parece sensato abrigar la sospecha (creada fundamentalmente por una impresión cualitativa al realizar las grabaciones) de que esta novedad afecta diferencialmente más a los sujetos normales, y en especial a los mayores, por su capacidad de comprensión de lo inusual (respecto a las actividades cotidianas del centro) de esta situación de interacción. Esto sería una causa posible de un fenómeno, observado especialmente en el grupo de normales, indirectamente confirmado por los resultados y que hemos denominado «inhibición sumisa».

De esta forma, el análisis de la conducta social de los sujetos de este estudio refleja las diferencias entre los grupos en cuanto a su comportamiento en una situación de interacción con un adulto "casi»" extraño. Pero muy probablemente no refleja toda la distancia y diferencia real que puede haber en la conducta social de los sujetos autistas respecto a los normales y los deficientes no autistas.

Por otro lado, todos y cada uno de los sujetos del grupo de deficientes y del grupo de autistas llevaban al menos seis meses de tratamiento psicopedagógico. Aunque esto no plantea problemas para la comparación entre grupos (pues ambos se encuentran en la misma condición) ni tampoco en la comparación con otras investigaciones; si matiza las conclusiones de nuestro estudio. Si bien es cierto que no abunda la aplicación de programas especificamente orientados a la mejora de la interacción social, también es cierto que los sujetos suelen mejorar en este terreno al recibir atención terapéutica general. Una de las múltiples razones de este efecto positivo indirecto es el entrenamiento en habilidades basicas (v. gr., contacto ocular) que constituyen prerrequisitos para aprendizajes especificos, pero que están también inevitablemente implicados en la interacción social.

Si esto es así, lo que aparece reflejado en los datos podria ser bien las conductas más caracterizadoras y específicas del comportamiento de los sujetos autistas en una situación de interacción o bien ( $y$ esto puede ser importante tenerlo en cuenta) 
las facetas de la conducta social de los sujetos autistas más resistentes a la intervención terapéutica.

De cualquier forma, creemos que las precauciones que se han tomado respecto al control experimental del trabajo y el alto número y amplitud de variables dependientes estudiadas, nos permiten extraer algunas conclusiones válidas para el estudio de este tema:

I. Si recordamos los resultados, son dos las variables dependientes en las que aparecen diferencias significativas y las dos hacen referencia a conducta visual: mira a la cara (sólo) y coorientación visual.

De alguna forma, los resultados de este estudio son comunes a los de las investigaciones de Hutt y Ounsted (1970). Estos autores consideran que la conducta social de los niños autistas no se diferencia morfológicamente de la de otros niños, salvo por la ausencia de fijación visual. No podemos considerar, sin embargo, que nuestro estudio venga a corroborar (aunque tampoco a contravenir) la hipótesis de la aversión visual, ni del «overarousal» como su causa, propuesta por Hutt y Ounsted (1970), ya que la anomalía visual manifestada por los niños autistas de esta investigación no se reduce al contacto ocular, sino que afecta también a la coorientación visual (categoría original de este estudio), esto es, a la atención compartida con el otro.

Por otro lado, también existen coincidencias con los trabajos de otros autores, cuyo marco teórico explicativo es distinto. Nos estamos refiriendo a autores que podriamos considerar representantes (entre otros) de las teorías cognitivo-organicistas. Asi, por ejemplo, Hermahn y O'Connor (1970-1983) consideran que la alteración fundamental del autismo consiste en un déficit central que afecta a la codificación e integración intermodal, lo que deteriora severamente toda conducta compleja, incluida la interacción social (O'Connor y Hermelin, 1983).

En su artículo de 1967, O’Connor y Hermelin publican los resultados de una serie de experimentos con medida de la fijación visual de sujetos autistas, deficientes y normales. Los datos indican que los niños autistas, al igual que los otros, prefieren la imagen de una cara, un estímulo nuevo frente al habitual... y que miran por igual una cara con los ojos abiertos que si los tiene cerrados. Pero en los tres experimentos que exponen, se manifiesta una cierta anomalía en la conducta visual de estos niños: o bien miraban cada fotografía menos tiempo que los sujetos de los grupos control o pasaban más tiempo mirando el fondo de la caja negra utilizada.

En definitiva, dejando a un lado las divergencias de hipótesis explicativas, existe un acuerdo en la mayoría de los autores (y este estudio viene a sumarse) sobre la existencia de una anomalia en la conducta visual de los niños autistas. Este punto confirma el interés de incluir la fijación visual como uno de los objetivos de observación, siendo ya una de las variables más utilizadas en los estudios sobre la responsividad social de los niños autistas.

Vista la importancia de la mirada a la cara y la coorientación visual como índices discriminativos entre los grupos de sujetos utilizados en este estudio, entendemos que sería necesario continuar en la profundización del tema fundamentalmente en dos líneas, entre otras muchas posibles:

I. Análisis exhaustivo y reflexivo de investigaciones anteriores que estudien el papel que desempeña la atención visual en el desarrollo social y comunicativo de la persona (v. gr. Bruner, I977).

2. Aplicación y creación de sistemas de observación y valoración de dichas conductas que vayan más allá del análisis de frecuencia y duración. Sistemas que permitan entrar en una valoración funcional del grupo de conductas implicadas en lo que denominamos "atención» visual, y que, al mismo tiempo, proporcionen información de cómo se asocian este tipo de conductas con otras, en el funcionamiento real del sujeto.

En esta línea, en nuestro trabajo se ha estudiado separadamente la frecuencia global de miradas a la cara y la de aquellas miradas a la cara que no aparecen asociadas a conductas comunicativas o expresivas, cuya finalidad se interpreta de mantenimiento del contacto y fijación de la atención en el otro, como referencia que es en una situación de interacción. Además, incluye la observación de la atención visual compartida, por medio de la categoría de coorientación visual. Resulta muy sugerente que sean precisamen- 
te estas dos las categorías en las que se manifiestan diferencias significativas entre los grupos. Pero esto no supone más que una ligera aproximación y un aliciente para lo que podria ser un estudio específico y amplio en la línea que acabamos de señalar.

II. En segundo lugar, querríamos comentar uno de los aspectos, a nuestro juicio, más interesantes de los resultados de esta investigación: las variables de conducta en que aparecen diferencias significativas entre los grupos son siempre de aproximación e interactivas.

$\mathrm{Al}$ analizar las conductas en tres grandes bloques, el nivel inferior de los autistas en las de «aproximación e interactivas» se manifiesta no sólo respecto a los niños normales, sino también respecto a los deficientes no autistas, aumentando, por tanto, las posibilidades de atribuir esta inferioridad de nivel al autismo infantil precoz.

Sin embargo, no aparecen diferencias entre los grupos en cuanto a conductas de evitación $\mathrm{y} / \mathrm{o}$ escape, ni global ni aisladamente consideradas.

Estos datos entran en discrepancia con los resultados de las investigaciones de Richer (1976) y fundamentalmente con algunas de sus conclusiones: «los niños autistas actúan para reducir la probabilidad de encuentros sociales" y "se alejan más de las personas"y.

Varias razones pueden dar cuenta de esta discrepancia:

1. Dejando a un lado la no equivalencia real de las categorias de observación, la situación de observación sí es claramente diferente. I a investigación de Richer (1976) se desarrolla en el patio de recreo y las interacciones tenian lugar fundamentalmente entre pares (autistas $y$ deficientes no autistas). Nuestro estudio, sin cmbargo, observa la interacción del niño con un adulto no familiar.

2. Fiste autor sólo utilizó como criterios de igualación entre el grupo de autistas y el de deficientes la edad cronológica y el sexo. Eligió luego sujetos normales de edades cronológicas inferiores coherentemente con la idea de que el desarrollo mental de los otros dos grupos era inferior al de su edad cronológica.

En nuestro trabajo, los sujetos autistas y los deficientes no autistas están igualados uno a uno en sexo, edad cronológica y edad mental y, por tanto, en cociente de desarrollo, están, asimismo, igualados con los sujetos normales en sexo y edad mental.

3. Richer registra como conductas de escape, considerando como tales ciertos movimientos de cabeza y cuerpo, alejamientos, ciertas muecas faciales... a través del análisis del comportamiento del sujeto en situaciones en las cuales no existen garantías constantes de que esos movimientos sean tal evitación o escape.

En el trabajo que nos ocupa, sólo se registran tales conductas en los momentos de la sesión de interacción en los cuales la intervención del adulto en la conducta del niño era patente $y$ por tanto, la causa más probable de su conducta.

Aun así, se podría argüir que la ausencia, en nuestros resultados, de diferencias significativas en cuanto a conductas de evitación puede ser atribuible al efecto de la intervención terapéutica en los sujetos (en la medida en que pueda haber incidido en la extinción o disminución de dichas conductas). Ahora bien, como ya habíamos comentado, no parece que nuestro trabajo muestre, en este aspecto, grandes diferencias con otras investigaciones, ya que en ninguna de ellas se señala que se hayan utilizado como sujetos experimentales niños que no hubiesen recibido o estuviesen recibiendo tratamiento psicopedagógico, $y$, de hecho, creemos, por múltiples razones, que es poco probable que haya ocurrido asi. Por tanto, nuestros resultados son perfectamente comparables con los de otras investigaciones y suficientemente sólidos como para obtener conclusiones: entonces, diremos que nuestros datos no confirman la idea de que uno de los aspectos más característicos de la conducta social de los niños autistas sea la evitación activa de interacción o encuentros sociales, sino más bien se acercan a un planteamiento explicativo (de la conducta social anómala de dichos niños) con base en una incompetencia interactiva.

III. Es evidente que los trabajos de Hutt y Ounsted y los de Richer y colaboradores resultan altamente sugerentes para cualquier estudioso del tema, en cuanto que sitúan sus observaciones en un plano que intenta dar una «explicación funcional» de los fenómenos estudiados. 
Nuestra opinión, sin embargo, es que una "explicación funcional» requiere un método global de observación y análisis de las conductas sociales más elaborado y cualitativamente distinto que el utilizado tanto por estos autores como en nuestro trabajo.

En este sentido, entendemos que una definición de las conductas, no sólo por sus características topográficas, sino por su valor funcional en el momento en que se produce, podria aportar información de gran interés para la comprensión de la conducta social en general y la de los niños autistas en particular. Asi, por ejemplo, la conducta de "señalar» («extiende y orienta en una dirección específica el/los brazos/s o el dedo índice») puede servir funcionalmente tanto para compartir la atención del adulto por medio de un objeto, como para pedirlo. Por tanto, una mera descripción en términos topográficos parece insuficiente para explicar el valor real de esta conducta en una situación interactiva especifica.

\subsection{Resultados y discusion: parte $I I$}

Un análisis minucioso e individualizado de los resultados obtenidos nos llevó a plantearnos con detenimiento el posible efecto de la variable edad mental sobre la conducta social diferencial de los sujetos en la situación de interacción. El estudio del efecto de esta variable se nos aparece como una interesante línea de investigación sobre el tema, que puede tener implicaciones comprensivas, diagnósticas y metodológicas útiles.

Por ello, se pensó en la posibilidad de hacer un nuevo análisis de los datos, en la medida que fuese viable, a fin de que sirviera de tanteo o pilotaje previo a una investigación en profundidad y para satisfacer provisionalmente nuestra curiosidad científica.

En concreto, los datos y la bibliografía utilizada daban pie a intentar comprobar estadísticamente si distintos niveles de edad mental implicarian mayor o menor número de variables dependientes en las que los grupos diagnósticos presentan diferencias significativas, y si, por otro lado, dentro de cada grupo diagnóstico se encuentran diferencias significativas en 92 ciertas pautas de interacción social en función del nivel de edad mental de los sujetos.

Para ello, se introdujo en el estudio otra variable independiente (edad mental) que se operativizó en dos niveles: I) sujetos con edad mental inferior a veinticuatro meses, y 2) sujetos con edad mental superior o igual a veinticuatro meses. De esta forma surgieron seis grupos de N.S. iguales (cinco sujetos en cada condición).

Dado que las variables dependientes utilizadas no presentaban características paramétricas, no fue posible obtener un indice de interacción de las variables independientes (grupo diagnóstico y edad mental).

Los resultados, en sus valores numéricos, y el procedimiento estadístico utilizado se encuentran detallados en las tablas VI, VII, VIII, IX, X y XI.

Como podemos observar, estos datos numéricos y la consulta a las tablas de puntuaciones medias de los grupos (que no se especifican en este artículo) indican, dicho muy esquemáticamente, lo siguiente:

1. En cuanto a la comparación de los tres grupos diagnósticos:

I. I. Al comparar los cinco sujetos autistas de edad mental inferior a 24 meses, con los deficientes y normales que les corresponden por igualación de sujetos, vemos que:

- Ios sujetos autistas presentan un nivel de contacto físico pasivo mantenido inferior al de los sujetos normales y al de los sujetos deficientes no autistas.

- También presentan un nivel significativamente menor que los normales en miradas a la cara del adulto (tanto en la puntuación global como cuando sólo se evalúan las no asociadas a otras conductas comunicativas o expresivas).

- Además, los sujetos autistas presentan una frecuencia de contactos físicos activos y de sonrisa, significativamente menor que los sujetos deficientes no autistas.

1.2. Sin embargo, al comparar los cinco sujetos autistas con edad mental superior o igual a 24 meses y los sujetos normales y deficientes igualados, no apa- 
Aplicación de Anova no paramétrico (Kruskal-Wallis) de un criterio de clasificación por rangos para tres grupos, para la comparación de sujetos autistas, deficientes $y$ normales de edad mental inferior a veinticuatro meses

\begin{tabular}{|c|c|c|}
\hline Variable & $\begin{array}{l}\text { Estadistico } \\
\text { empirico }\end{array}$ & $\begin{array}{l}\text { Nivel de } \\
\text { signif. }\end{array}$ \\
\hline Proximidad . . . . . . & 0.15636 & .9248 \\
\hline Contacto físico pasivo. & 8.76853 & $.0125^{*}$ \\
\hline Contacto físico activo. & 7.23290 & $.0265^{*}$ \\
\hline Desplazamientos hacia. & 0.79204 & .6730 \\
\hline Se vuelve hacia ...... & 0.79635 & .67 is \\
\hline Vuelve cabeza hacia . . & 3.45230 & .1780 \\
\hline Mira al $\ldots \ldots \ldots \ldots$ & 2.01659 & .3648 \\
\hline Mira cara (sólo) . . . . & $.7 .7399^{6}$ & $.0209^{*}$ \\
\hline Mira cara (global). & 7.21999 & $.0271 *$ \\
\hline Coorientación visual. & 6.26326 & $.0436 *$ \\
\hline Sonrisa . . . . . . . & 1.46833 & .4799 \\
\hline Abrazo $\ldots \ldots \ldots$ & 1.54060 & .4629 \\
\hline Tira de $\ldots . . \ldots \ldots$ & 4.28571 & .1173 \\
\hline Reproduce $\ldots \ldots \ldots$ & 0.42818 & .8073 \\
\hline Comunicación oral ... & 2.06649 & .3559 \\
\hline Señala $\ldots \ldots \ldots \ldots$ & .1 .16715 & .5579 \\
\hline Muestra/da . . . . . . & 0.31727 & .8533 \\
\hline Vocalizaciones ...... & .0 .63636 & .7275 \\
\hline Coge $\ldots \ldots \ldots \ldots$ & 0.15269 & .9265 \\
\hline Acude llamada ...... & . 2. 52984 & .2823 \\
\hline Se aleja ......... & 0.46578 & .7922 \\
\hline Se vuelve de ..... & 0.40531 & .8166 \\
\hline Vuelve cabeza de... & 2.04098 & .3604 \\
\hline Cara pared ........ & .2 .16406 & .3389 \\
\hline Cabeza baja . . . . . . & .3 .74653 & .1536 \\
\hline L.lantos/gritos . . . . . & 4.48420 & .1062 \\
\hline Aparta al adulto ..... & .2 .47174 & $.209^{6}$ \\
\hline Cierra ojos $\ldots \ldots \ldots$ & .2 .03354 & .3618 \\
\hline Se tapa ojos/oidos . . & .4 .86486 & .0878 \\
\hline Se protege $\ldots \ldots \ldots$ & 0.04082 & $.979^{8}$ \\
\hline Comunicacion oral ( $\mathrm{E}$ ). & 4.28571 & .1173 \\
\hline Agresiones al entorno. & .2 .03354 & .3618 \\
\hline Agresiones al adulto. & 2.00000 & .3679 \\
\hline Autoagresiones . . . . . & . 2.96114 & .2275 \\
\hline
\end{tabular}

${ }^{*} \mathrm{p}(x) \leqslant ; 0,05$.
Aplicación de Anova no paramétrico de un criterio de clasificación por rangos para tres grupos, para la comparación de autistas, deficientes y normales de edad mental superior 0 igual a 24 meses.

\begin{tabular}{|c|c|c|}
\hline Variable & $\begin{array}{l}\text { Estadistico } \\
\text { empirico }\end{array}$ & $\begin{array}{l}\text { Nivel de } \\
\text { signif. }\end{array}$ \\
\hline Proximidad . . . . . . & 2.2199 & .3296 \\
\hline Contacto físico pasivo. & 0.1974 & 9060 \\
\hline Contacto físico activo. & 5.1978 & .0744 \\
\hline Desplazamientos hacia. & 2.8356 & .2422 \\
\hline Se vuelve hacia ...... & 0.7439 & .6894 \\
\hline Vuelve la cabeza hacia & 1.8311 & .4003 \\
\hline Mira al $\ldots \ldots \ldots \ldots$ & 2.0021 & .3675 \\
\hline Mira cara (sólo) ..... & I. 1399 & .3655 \\
\hline Mira cara (global) ... & 1.0085 & .6039 \\
\hline Coorientación visual . & 0.4230 & .8094 \\
\hline Sonrisa . . . . . . . & 0.5409 & .7630 \\
\hline Abrazo ......... & 2.2992 & .3168 \\
\hline Tira de ....... & 2.0335 & .3618 \\
\hline Reproduce . . . . . . & 2.8253 & .2435 \\
\hline Comunicación verbal. & I. 4888 & .4750 \\
\hline Señala $\ldots \ldots \ldots \ldots$ & 3.9725 & .1372 \\
\hline Muestra/Da ....... & 1.5753 & .4549 \\
\hline Vocalizaciones ...... & 1.0769 & .5836 \\
\hline Coge ......... & 1.9645 & .3749 \\
\hline Acude llamada . . & 5.5351 & .0628 \\
\hline Se aleja $\ldots . . .$. & 1. 5492 & .4609 \\
\hline Se vuelve de $\ldots \ldots$ & 1.0953 & .5783 \\
\hline Vuelve la cabeza de . . & 0.0408 & $.979^{8}$ \\
\hline Cara a la pared ...... & 2.0000 & .3679 \\
\hline Cabeza baja .... & 2.0335 & .3618 \\
\hline Llantos/gritos ..... & 1.7187 & .4234 \\
\hline Aparta al adulto ..... & . 2.0000 & .3679 \\
\hline Cierra los ojos . . . . . & . 3.3471 & .1876 \\
\hline Se tapa ojos/oidos ... & . 2.2992 & .3168 \\
\hline Se protege $\ldots \ldots \ldots$ & 1.4221 & .4911 \\
\hline Comunic. verbal (E) . & 2.0000 & .3679 \\
\hline Agresiones al entorno. & 2.6466 & .2662 \\
\hline Agresiones al adulto. & 2.0000 & .3679 \\
\hline Autoagresiones . . . . . & . 1.1399 & .5659 \\
\hline
\end{tabular}

los niveles de significacion no permiten rechazar la hipótesis nula de que no existen diferencias entre los grupos, para ninguna variable (p. (x) $\leqslant .05)$.

\section{TABIA VII}

Prueba de rangos de $U$ de Mann-Whitney para la comprobación de la significación entre cada par de grupos (Autistas-Normales; Autistas-Deficientes; Deficientes-Normales; todo ellos con edad mental inferior a veinticuatro meses)

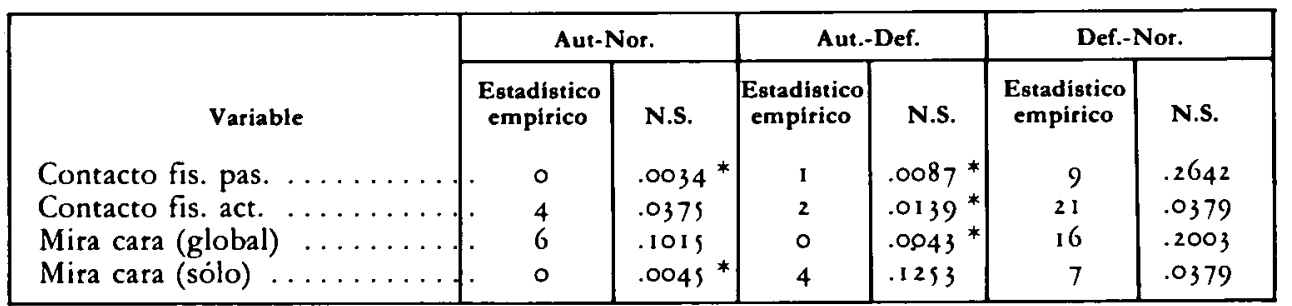


Aplicación de la prueba de rangos $U$ de Mann-Whitney para la comparación entre dos grupos: los dos grupos: los dos niveles de edad mental dentro de cada grupo diagnóstico.

\begin{tabular}{|c|c|c|c|c|c|c|}
\hline \multirow[b]{2}{*}{ Variable } & \multicolumn{2}{|c|}{ Autistas } & \multicolumn{2}{|c|}{ Deficientes } & \multicolumn{2}{|c|}{ Normales } \\
\hline & $\begin{array}{l}\text { Estadistico } \\
\text { empítico }\end{array}$ & N.S. & $\begin{array}{l}\text { Estadistico } \\
\text { empirico }\end{array}$ & N.S. & $\begin{array}{c}\text { Estadístico } \\
\text { empirico }\end{array}$ & N.S. \\
\hline Proximidad . . . . & 8 & .1729 & 11 & .3770 & 16 & .2010 \\
\hline Contacto fis. pas. & 25 & $.0035 *$ & 13 & .4583 & 13 & .2010 \\
\hline Contacto fis. act. . . . . . & 20 & .0569 & 9 & .2648 & 2 & $.0178^{*}$ \\
\hline Desplazamientos hacia ... & I 2 & .5000 & 3 & $.0226 *$ & II & .3756 \\
\hline Se vuelve hacia ...... & 12 & .5000 & $s$ & .0712 & is & .2636 \\
\hline Vuelve la cabeza hacia & 8 & .1973 & 10 & .3371 & 12 & .4582 \\
\hline Mira al $\ldots \ldots \ldots \ldots \ldots$ & 17 & .1729 & 16 & .2317 & 12 & $.458 \mathrm{I}$ \\
\hline Mira cara (sólo) . & 21 & $.0379^{*}$ & 17 & .1736 & 13 & $: 4170$ \\
\hline Mira cara (global) .. & 20 & .0586 & is & .3008 & 15 & .3002 \\
\hline Coorientación visual & 21 & $.0361 *$ & 12 & .5000 & 16 & .2296 \\
\hline Sonrisa $\ldots \ldots \ldots \ldots$ & 17 & .1448 & 11 & .4115 & 8 & .1546 \\
\hline Abrazo . . . . . . . . & is & .2603 & 7 & .1188 & 7 & .0680 \\
\hline Tira de ..... & is & .1987 & 12 & .4531 & 12 & .9000 \\
\hline Reproduce ...... & 20 & $.0468 *$ & 21 & $.0330^{*}$ & 19 & .2512 \\
\hline Comunicación verbal .... & is & .2603 & 17 & .1580 & 12 & .9000 \\
\hline Señala $\ldots \ldots \ldots \ldots$ & 10 & .1587 & 14 & .3729 & 12 & .4531 \\
\hline Muestra/Da $\ldots \ldots \ldots$ & 12 & .4572 & 16 & .1943 & 10 & .3327 \\
\hline Vocalizaciones & is & .2564 & 12 & .9000 & 19 & .1987 \\
\hline Coge ........ & 18 & .0774 & 12 & .9000 & 13 & .4549 \\
\hline Acude llamada & 10 & .3367 & 12 & .4572 & 7 & .0680 \\
\hline Se aleja $\ldots \ldots \ldots \ldots$ & 11 & .3767 & is & .2887 & 10 & .2991 \\
\hline Se vuelve de ...... & 7 & .1187 & 13 & .4155 & 7 & .1379 \\
\hline Vuelve la cabeza de & 12 & .5000 & II & .3493 & 19 & .1987 \\
\hline Cara a la pared ....... & 7 & .0680 & 12 & .4408 & 12 & .5000 \\
\hline Cabeza baja .......... & 2 & $.0091 *$ & 19 & .2193 & 8 & $.144^{8}$ \\
\hline Llantos/gritos . . . . . . & 8 & .1995 & 14 & .3752 & 17 & .1448 \\
\hline Aparta al adulto ........ & 10 & .3028 & 12 & .5000 & 10 & .1987 \\
\hline Cierra los ojos ........ & 16 & .2042 & 14 & .3190 & 12 & .9000 \\
\hline Se tapa ojos/oídos ..... & 1s & .2193 & 7 & .1188 & 12 & .5000 \\
\hline Se protege $\ldots \ldots \ldots \ldots$ & is & .2193 & 20 & $.0468 *$ & 17 & .1439 \\
\hline Comunicación verbal (E) ... & 7 & .0680 & is & .1587 & 12 & .5000 \\
\hline Agresiones al entorno ..... & 12 & .4408 & 14 & .3190 & 12 & .5000 \\
\hline Agresiones al adulto ........ & 10 & .1587 & is & .1987 & 12 & .5000 \\
\hline Autoagresiones ......... & 13 & .4165 & 17 & .1736 & 21 & $.0370^{*}$ \\
\hline
\end{tabular}

En la tabla aparecen señaladas con un asterisco las variables para las cuales es posible rechazar la hipotesis nula de que no existen diferencias significativas entre los grupos, considerando un $p$. $(x) ! \leqslant 10 \%$.

recen diferencias estadisticamente significativas en ninguna de las 34 variables analizadas.

2. En cuanto a la comparación de los dos niveles de edad mental dentro de cada grupo diagnóstico:

2.1. Al comparar los cinco sujetos autistas de edad mental inferior a 24 meses y los cinco autistas de edad mental superior o igual a 24 meses, vemos que:

- Los sujetos autistas de edad mental cativamente menor que los de edad mental superior, en las variables de contacto físico pasivo mantenido, miradas a la cara del adulto (sólo), sonrisa y comunicación verbal.

- Y, sin embargo, los de edad mental inferior presentan un nivel significativamente más elevado que los de edad mental superior en llantos y gritos.

2.2. Los sujetos deficientes de edad mental inferior a 24 meses presentan:

- Un nivel significativamente menor que los deficientes con edad mental 
superior a 24 meses, en comunicación verbal (tenga o no su contenido carácter de evitación).

- Sin embargo, el nivel de desplazamientos hacia el adulto es más elevado que en los sujetos con edad mental superior o igual a 24 meses.

2.3. En lo que se refiere a los sujetos normales:

- El nivel de coorientación visual presentado por los normales de edad mental inferior, resulta significativamente menor que el de los sujetos normales de edad superior o igual a 24 meses.

- Sin embargo, la frecuencia de contacto físico activo de los sujetos con edad mental inferior a 24 meses es significativamente mayor que la de los sujetos de edad mental superior.

De estas nuevas comparaciones resulta especialmente llamativo que:

a) Al reducir la muestra a los cinco sujetos autistas de edad mental inferior a 24 meses y sus igualados deficientes y normales, aumenta el número de variables en las que los sujetos autistas presentan un nivel significativamente menor que los otros grupos, y se distancian más de las puntuaciones del grupo de deficientes.

b) Sin embargo, al comparar los cinco sujetos autistas de edad mental superior o igual a 24 meses no aparecen diferencias significativas estadísticamente entre los grupos en ninguna variable.

c) Al realizar comparaciones entre los sujetos de edad mental inferior a 24 meses y sujetos de edad mental superior o igual a 24 meses dentro de cada grupo diagnóstico, son los autistas los que presentan mayor número de diferencias significativas en las variables conductuales mediadas en la situación de interacción. $Y$ estas diferencias indican un menor nivel y frecuencia de conductas de aproximación o interactivas cuando los sujetos tienen una edad mental inferior a 24 meses.

Globalmente, estos resultados presentan cierta coherencia con estudios de seguimiento de niños autistas, que han demostrado que pueden ocurrir cambios al aumentar la edad (De Myer, et al., 1973; Rutter Kanner, et al., 1972; Rutter, 1970). Estos estudios indican que, aunque algunos niños no hacen ningún progreso y unos pocos se deterioran, un porcentaje considerable se vuelve menos resistente al cambio y pierden indiferencia hacia las personas. Lorna Wing (197I) señala que las señales de desapego e indiferencia son, especialmente, claras en los menores de cinco años.

Por otro lado, la relación de los distintos niveles intelectuales y las alteraciones cognitivas en el autismo ha sido ampliamente estudiado (vid. revisión Prior, I 984), y actualmente es reconocido como un factor clave en la discusión sobre la clarificación y comprensión del autismo, la comparación y reflexión de investigaciones y la búsqueda de criterios para el diagnóstico diferencial.

Este trabajo, por su parte, resalta la necesidad de investigación de las variables edad de desarrollo y cociente de desarrollo y su relación con la conducta social de los niños autistas.

Sería necesario, además, cuestionarse hasta qué punto es correcto mantener criterios diagnósticos para el «síndrome de autismo infantil precoz» que partan de índices de conducta claramente observables en niños con edades mentales inferiores a 24 meses, pero bastante menos intensos en niños de nivel superior. Aunque, como ya señaló Rutter (1978), el 75 por 100 de los sujetos autistas pueden definirse intelectualmente como «deficiente mental", parece arriesgado contar únicamente con sistemas diagnósticos que sólo parecen «altamente fiables» para niveles de desarrollo bajos.

\section{CONCLUSIONES}

La realización del presente estudio comparativo de la conducta social de niños autistas deficientes y normales, en situación de interaćción, lejos de desalentar, nos ratifica en nuestro planteamiento inicial, en principio, en dos aspectos concretos:

Por una parte, la utilización de un grupo de niños normales y el diseño de sistemas de observación y análisis aplicables a este tipo de sujetos nos ha permitido descubrir hasta qué punto resultaria interesante la investigación rigurosa sobre aspectos concretos que parecen fundamentales, tanto para la comprension del desarrollo social del niño, como para la formacion de un "modelo» de desarro- 
llo social normal, que sirva de base para el estudio de grupos infantiles patológicos.

Por otro lado, la comparación de sujetos autistas y deficientes no autistas sirve, en nuestra opinión, para resaltar el interés del estudio de las pautas de interacción social en la investigación psicológica de grupos de niños con alteraciones severas del desarrollo.

Desde otro punto de vista, este estudio sirve de base para indicar algunos caminos posibles de investigación futura. Se plantea la necesidad de un estudio minucioso por rangos de edad que proporcionará una información más exacta de las diferencias y peculiaridades de la conducta social de los sujetos autistas, frente a los normales y deficientes igualados, y que tendría serias implicaciones para el diagnóstico.

Ya, por último, expresar que la metodología de investigación utilizada en este trabajo (y la mayoria de las que aparecen en la literatura revisada) se nos manifiesta, a pesar de sus aportaciones y mejoras, como insuficiente y limitada. Insuficiente para recoger toda la información que puede dar la observación minuciosa de la interacción social y limitada para dilucidar los trastornos y características que diferencian la conducta social de los sujetos autistas de la de otros grupos, normal o patológicos. Una observación completa de lo que parece ser la información relevante en el estudio de la interacción infantil no parece depender sólo de la complejidad y amplitud del estudio según el número de categorías de observación, y su capacidad representativa de habilidades realmente implicadas en la interacción social. Consideramos que el progreso en el estudio de este tema vendria dado, a partir de ahora, por estudios complementarios que tengan en cuenta estos aspectos, pero que pasen a un nivel de análisis de la conducta diferente. El análisis por funcionalidad de la conducta, o por la vinculación real de conductas entre sí en la actuación del sujeto (el estudio de secuencias completas de conducta), o por la adecuación de la conducta del sujeto a la de su compañero de interacción (me diante el estudio de secuencias interactivas...), pueden constituir métodos que convendría explorar y poner en funcionamiento en trabajos posteriores.

\section{Resumen}

El presente articulo expone una investigacion cuyo objetivo es estudiar comparativamente la conducta de los niños autistas, deficientes no autistas y normales, en una situación de interacción diadica con un adulto. La muestra estaba compuesta por 30 niños, 10 en cada grupo. Los niños de los distintos grupos diagnósticos estaban igualados uno a uno en edad mental y sexo. Los deficientes y los autistas fueron igualados además en edad cronológica. Cada sujeto fue grabado mediante video en una sesión de interacción social. La conducta de los sujetos se codificó atendiendo a 34 categorias de observación que recogian distintos tipos de conducta (conductas proxémicas, de atención, expresivas, instrumentales, estrategias de comunicación, conductas de buida, agresivas...). Se discuten los resultados y sus implicaciones, sugiriendo nuevas lineas de investigación.

\section{Summary}

The aim of this research is the comparative study of autistic, mentally retarded non-autistic and normal children, in a diadic situation interaction with an adult. The sample includes 30 children, 10 cbildren, matched in Mental Age and sex in each group. Retarded and autistic children were paired as well by cbronological age. Each subject was vides recorded during a social interaction session. Behavior of subjects was coded in 34 observing categories stating different types of bebavior - proxemic, attention, expresive, instrumental, escape agressive behaviors, communication strategies... Results and implications are discussed and new lines of research suggested.

\section{Résumé}

Dans cet article on expose une recbercbe dont le but est l'étude comparatif de la conduite des enfants autistiques, des enfants déficients et des enfants normaux dans une situation d'interaction diadique avec un adulte. L'échantillon était constitué par 30 enfants, so par groupe. Les enfants de ces trois groupes étaient appareillés en áge mentale et sexe. Les déficients et les autistiques furent aussi appareillés en âge réel. Chaque enfant a eté enregistré au vidéo au cours d'une séance dinteraction sociale. La conduite des enfants fut codifiée par rapport à 34 catégories d'observation contenant des différents types de comportements (proxémiques, dattention, expresives...). Les résultats et leurs conséquences sont discutés, avec des suggérences de nouvelles lignes de recbercbe. 
Bi.ACK, M.; Freeman, B. J., y MONTGomery, J.: "Systematic observation of play behavior in autistic children". Journal of Autism of Childhood Schizophrenia, , (4), págs. $363-371$, 1975 .

BRUNER, J. S.: "Early social interaction and language acquisition". En, H. R. SCHAFFER (Ed.): Studies in motber-infant interaction. Londres, Academic Press, págs. 271-290, 1977.

CANTwel.l, D. P.; BAkER, L., y Rutter, M.: «Families of autistic and dysphasic children: I Family life and interaction patterns». Archives of General Psychiatry, 36 (6), págs. 682-687, 1979.

Clancy, H. D., y Rendle-Short, J.: "The diagnosis of infantile autism». Developmental Medicine Child Newrology, $11,1969$.

Coleman, M.: The autistic syndromes. Nueva York, Elsevier, 1976.

Creak, et al.: "Schizophrenic syndrome in childhood». Developmental Medicine Child Neurology, 6, págs. \$30-\$35, 1964 .

DEMYER, M. K. et al.: "Prognosis in autism: A follow-up study». Journal of Autism Cbildbood Scbizopbrenia, 3, págs. 199-246, 1973 .

DSM III: Diagnosis and Statistical Manual of the American Psychiatric Association. 1980.

FrefmAN, B. J. et al.: «Behavior Observation Scale: preliminary analysis of the similarities and differences between autistic and mentally retarded children". Psychological Reports, 44, págs. 519-524, 1979.

HERmEl.iN, B., y O'CONNOR, N.: "The response and self-generated behavior of severely disturbed children and severely subnormal controls». Brit. J. Soc. Clin. Psychol., 2, págs. 37-43, 1963.

Hermiji.in, B. y O'Connor, N.: Psychological experiments with autistic children. Londres, Pergamon Press, 1970.

HUT"T, C. y OUNSTF.D, C.: "The biological significance of gaze aversion with particular references to the syndrome of childhood autism». Behavioral Science, 11, págs. 346-396, 1966.

HutT, C. y OUNSTI:D, C.: "Gaze aversion and its significance in childhood autism». Fn HLTT yLTT (Eds.): Behavior studies in Psychiatry. Oxford, Pergamon, 1970.

HUTT, C. y VAIZEY, M. J.: «Differential effects of group density on social behavior». Nature, 209, págs. $1371-1372,1966$.

KANNER, I.: «Autistic disturbances of affective contact». Nervous Cbild, 2, págs. 21 7-250, 1943.

KANNER, L. et al.: "How far can autistic children go in matters of social adaptation?" Journal of Autism and Childbood Scbizophrenia, 2, págs. 9-33, 1972.

Krug, D. A.; ARICk, J. R., y Almond, P. J.: “Autism Behavior Checklist (A.B.C.). En D. A. KRLG; J. R. ARICK y P. J. AIMONI): Examiner's manual: autism screening instrument for educational planing. ASIEP. USA, Education Company, 1980.

LoRd, C.; SChopier, F. y Revicki, D.: “Sex differences in autism». Journal of Autism and Detelopmental Disorders, 12 (4), págs. $317-330,1982$.

MCHALE, S. M.: "Social interaction of autistic and non handicapped children during free play". American Journal of Orthopsychiatry, $53(1)$, págs. $81-91,19^{8} 3$.

O'CONNOR, N. y HERMEIIN, B.: "Measures of distance and motility in psychotic children and severely subnormal controls". Brit. J. Soc. Clin. Psycbol., 3, págs. 29-33, 1983.

O'CONNOR, N. y HERMEIIN, B.: "The selective visual attention of psychotic children". Journal of Child Psychology and Psychiatry, 8, págs. $167-179,1967$.

O'CONNOR, N. y HERMilin, B.: «Peripheral and central handicaps and encoding». Journal of Child Psychology' and Psychiatry and Allied Disciplines, 24 (1), págs. 39-48, 1983.

ORNIT\%, E. M.: "Childhood Autism: A review of the clinical and experimental literature". California Medicine, i 18 , págs. $21-47,1973$.

PRIOR, M.: "Developing concepts of childhood autism: the influence of experimental cognitive research». Journal of Consulting and Clinical Psychology, 52 (1), págs. 4-16, 1984.

RFICHIER, R. J. y SCH(OpI.F.R, F.: “(Observations on the nature of human relatedness". Journal of .Autism and Cbildbood Schizopbrenia, 1, págs. $283-296,1971$.

Riciler, J.: "The social-avoidance behaviour of autistic children». Animal Bebaliour, 24 (4), págs. 898-906, 1976.

RICHER, J y RICHARDS, B.: «Reacting to autistic children: The danger of trying to hard». British Journal of Psychiatry, 127, págs. 526-529, 1975.

Richir, J. y Coss, R. G.: "Gaze aversion in autistic and normal children". Acta Psycbiatrica Scandinatica, $\$ 3(3)$, págs. $193-210,1976$.

RIMi.AND, B.: "The differentiation of childhood psychosis: An analysis of checklists for 2.218 psychotic children". Journal of Autism and Childhood Schizophrenia, 1, págs. I 61-1 74, 1971.

Rırvo, F. R.: Autism: Diagnosis, current research and management". Nueva Y'ork. Spectrum Press, 1976.

Ritvo, F. R. y Freemin, B. J.: «National Society for Autistic Children's: definition of the syndrome of autism». Journal of Pediatric Psycbology, 4, págs. 146-148, 1977.

Rivièrt, A. y BtinCIION, M.: «Reflexiones sobre el lenguaje autista: I Análisis descriptivos y diferencias con la disfasia receptivas. Infancia y Aprendizaje, 13, págs. 89-120, 1981.

Rivitirt, A., et al.: Fscala para el diagnóstico de autismo. "Memoria de investigación de "evaluación y alteraciones de las funciones psicológicas en autismo infantil"'m, financiado por el CIDE. Madrid, 1984 .

Rogers, A. I. y FINF, H. J.: "Personal distance in play therapy with an autistic and a symbiotic psychotic child". Psychotherapy: Theory, Research and Practice, 14 (1), pags. $41-48,1977$.

RutTER, M.: "Autistic children: Infancy and adulthood». Seminars in Psychiarry, 2, págs. 435-450, 1970.

RUTTER, M.: "Diagnosis and definition of childhood autism". Journal of .1utism and Childbood Scbizophrenia, 8 , págs. $139-161,1978$.

SCHOPI.ER, E., et al.: "Toward Objective Classification of childhood autism: Childhood Autism Rating Scale (CARS)". Journal of Autism and Developmental Disorders, 10 (1), 1980.

STRAin, P. S. y CoOkE, T. P.: "An observational investigation of two elementary-age autistic children during free play". Psychology in the Schools, 13 (1), págs. 82-89, 1976. 
Strain, P. S.; KerR, M. M. y RAgl.AND, E. U.: «Effects of peer-mediated social initiations and prompting/reinforcement procedures on the social behavior of autistic childrenm. Journal of Autism and Developmental Disorders, 9 (1), págs. 41-\$4, 1979.

UCHIDA, I.: «Effects of interpersonal stimulation and interpersonal distance on social behavior in autistic children without EEG abnormalities and mentally retarded children with EE abnormalities". Japanese Journal of Child and Adolescente Psychiatry, 22 (5), págs. 367-368, 1981.

V.aN Dercar, D. H.; Powel.., S.; SANDI.ER, J. y VAN DeRCAR, C. M.: «Utilization of an experimentercontrolled manikin in analysis of social stimuli maintaning behavior of an autistic-like child". Psycbological Reports, 49 ( 1 ), págs. 47.57, 1979.

WING, L.: "Perceptual and language developmental in autistic children: A comparative study". En M. RUTTER (Ed.): Infantile autism: Concepts, characteristics and treatment. Londres, Churchill-Livingstone, págs. $173 \cdot 197,1971$. 